\title{
A Framework for Automatic Identification and Visualization of Mobile Device Functionalities and Usage
}

\author{
Renan H.P. Lima ${ }^{1}$, Moushir M. El-Bishouty ${ }^{2,3}$, and Sabine Graf ${ }^{2}$ \\ ${ }^{1}$ Federal University of Sao Carlos, Brazil \\ ${ }^{2}$ Athabasca University, Canada \\ ${ }^{3}$ City for Scientific Research \& Technological Applications, Egypt \\ renan.1ima90@gmail.com, \\ \{moushir.elbishouty, sabineg\} @athabascau.ca
}

\begin{abstract}
While mobile learning gets more and more popular, little is known about how learners use their devices for learning successfully and how to consider context information, such as what device functionalities/features are available and frequently used by learners, to provide them with adaptive interfaces and personalized support. This paper presents a framework that automatically identifies the functionalities/features of a device (e.g., Wi-Fi connection, camera, GPS, etc.), monitors their usage and provides users with visualizations about the availability and usage of such functionalities/features. While the framework is designed for any type of device such as mobile phones, tablets and desktop-computers, this paper presents an application for Android phones. The proposed framework (and the application) can contribute towards enhancing learning outcomes in many ways. It builds the basis for providing personalized learning experiences considering the learners' context. Furthermore, the gathered data can help in analyzing strategies for successful learning with mobile devices.
\end{abstract}

Keywords: Context modeling, visualization, mobile learning, device functionalities and their usage, personalization, ubiquitous learning analytics.

\section{Introduction}

The recent advances in mobile technologies have allowed the widespread use of mobile devices around the world for many different purposes. From educational point of view, learning can now take place anytime and anywhere using mobile devices (e.g., smartphones, tablets) to facilitate learner interaction and access learning contents with fewer restrictions of time and location [1-3]. Mobile settings bring important advantages for ubiquitous learning by providing a more flexible and authentic experience for learners.

The way people interact with devices is vital for their success. Looking at human computer interaction (HCI), it is apparent that interaction techniques are limited by 
the technology available [4]. However, due to the increasing diversity of users, technical systems and usage contexts, many aspects are relevant in understanding users' acceptance beyond the ease of using a system and the perceived usefulness [5]. HCI benefits from situational context modeling (such as location, surrounding environment and/or state of the device), as an implicit input to the system [4]; for example contextual information can be used to adapt the system input and output to the current situation (such as: font size, volume, brightness, privacy settings, etc.).

In ubiquitous environments, factors such as a learner's context influence the learning process in a mobile ubiquitous environment [2] and should be considered in order to provide appropriate support for learners. While mobile learning gets more and more popular, we do not know much about how learners use mobile devices for learning. Furthermore, context information in terms of what device functionalities/features are available and frequently used by learners is typically not considered when learners are presented with learning activities.

Data gathered from the usage of the learners' devices provide important information about the user and his/her context [6-7] and can, together with the availability of device functionalities/features, be used to build a context profile [3]. In this paper, we introduce a framework that aims at building a comprehensive context profile, containing information about the devices a learner uses, the available functionalities/features on a learner's device as well as how frequently the respective device functionalities/features are used by the learner. Such functionalities and features include, for example, internet connection types, existing sensors, camera, keyboard, touch screen and so on. The proposed framework enables a system to automatically identify, monitor and visualize the availability and usage of device functionalities/features in mobile devices and desktop computers.

While some ubiquitous and mobile systems use the devices simply as source of user's location data and do not consider different types of information that they can provide, other systems (e.g., Phone Usage [8], Elixir [9]) do not consider how the user interacts with his/her device for user profiling - i.e. which functionalities/features he/she prefers to use and when. Our framework differs from others since it considers the availability and the usage of device functionalities/features to build a comprehensive context profile.

By creating such a comprehensive context profile, the basis is built for providing personalized learning experiences to mobile learners. By knowing the advantages and limitations of a currently used device, learning activities can be tailored to available and frequently used device functionalities/features. For example, online videos are only suggested if appropriate internet connectivity is available. Furthermore, if the usage data show that the learner does not use the respective device for inputting large amounts of text, learning activities that require writing significant amounts of text are not recommended. On the other hand, if features such as a camera or GPS are detected, learning activities that require such features can be suggested. In addition, data about the usage of device functionalities/features provide valuable information about how learners actually use different devices for learning and provide insights into what types of learning activities can facilitate mobile learning. Moreover, such usage data can help in analyzing successful learning strategies in mobile learning. 
The paper is organized as follows: In section 2, related works are described. Section 3 introduces the proposed framework and Section 4 presents an application for Android phones developed based on the introduced framework. Section 5 concludes the paper.

\section{Related Work}

In the educational domain, the tracking and analyzing of device usage and the use of applications is particularly relevant and led to many educational institutions performing studies for this purpose. For example, the Educause Center for Applied Research (ECAR) has surveyed undergraduate students annually since 2004 about technology in higher education [10]. In 2012, ECAR collaborated with 195 institutions to collect responses from more than 100,000 students about their technology experiences. One of the key findings is that students want to access academic progress information and course material via their mobile devices [11]. Moreover, Curtin University [12] has presented a web survey since 2007, which was available to all students at the university for a two-week period through the student portal. The recent surveys [13] sought information on student access to the Internet off-campus, current and planned ownership and use of mobile devices, and perceptions as to how the learning experience at Curtin University might be enhanced with mobile devices, network services and online tools. Also, students were asked to report what they used their phones for (e.g., web access, SMS, MMS, and so on). The results of these surveys indicated that the majority of students were likely to have broadband (often wireless) access to the Internet off-campus, and use mobile devices, such as newer laptops and phones. The results also showed the familiarity of students with iTunes and iPhone apps (due to a high ownership of iPhones), and the ability to access wireless, take photos, send text (and some have the ability to record video and audio, and hold video conference) on a mobile device. Furthermore, the results indicated the ability to use Web 2.0 applications to create accounts, connect with others, communicate in web spaces, and indicate 'liking' and rating. Furthermore, Ally and Palalas [14] conducted a research study to determine the current state of mobile learning in Canada and to establish the direction Canada should take in the field of $\mathrm{m}$ learning. The study surveyed and interviewed small, medium, and large organizations from fifteen different sectors across Canada on their use of mobile learning and the future direction in that area.

From the abovementioned studies, it can be seen that mobile learning gets more and more popular. Student ownership and familiarity of mobile devices have increased over recent years, with most students reporting that they own a computer and/or a mobile phone [10]. The technologies themselves have also changed over the years, with increasing ownership of laptop computers and smart phones, corresponding to decreasing ownership of desktop computer and simple mobile phones[15][16].

With the increasing popularity of mobile learning, there is also a need for understanding how learners actually use their devices for learning. In addition to 
manually track device usage, systems were presented to automatically identify such information. Trinder [17], for instance, proposed a tool to automatically collect usage log data from personal digital assistants (PDAs) handheld devices used by students to access the university's virtual learning environment (VLE). The tool recorded in detail when PDA applications (including formative assessment applications) were being used and overcame a number of technical barriers in securing this information for later analysis. It was anticipated that such log information would provide reliable timing information regarding PDA use. Automatic logging was considered to be preferable in contrast to asking the participants to keep a diary of how and when they used the PDA. A manual diary method adds extra user overhead and unless the event is recorded contemporaneously it may be forgotten. Automatic logging also allowed the collection of additional information such as when the PDA was hot-synced to a desktop machine, or when the student used the "beam" facility to exchange items with another PDA. Patterns and modes of their PDA use - considering a range of factors including overall duration of use, use as a function of time of day or time of week, and the complexity of use (e.g. frequency of application switching within a usage session) - were obtained and correlated with exam results and access to the University's VLE. In addition to the logging data, selected cohorts of students were also subject to questionnaire and interview. Practical experiments were conducted to examine how students used the loaned PDAs and data visualization tools and techniques of data analysis were introduced to show the findings. The results indicated the benefits to students of general PDA use and specific use of the formative assessment quiz application. Another finding was that the bottlenecks to PDA use should be overcome to enhance its usage and student learning [18].

Moreover, several applications are developed in order to monitor the usage of smart mobile devices aiming at enchanting the device usage. Due to the advantages of open source, many of them are implemented for Android-powered mobile devices [19]. Android Status [20], for instance, features CPU memory usage, process list, mobile network information, Internet connections, network information, Wi-Fi Status, storage (i.e., SD card) usage, routing information, and others. Elixir [9] is another application with highly configurable widgets. It displays information about battery, CPU usage, memory usage, internal and external storage, display, Wi-Fi status, mobile network, location services (i.e., GPS), Bluetooth, sync, airplane mode, sensors, etc. CenceMe [21] is a different type of application, which not only tracks sensor-related information from users' mobile devices but also interprets this information in the context of social activities and allows sharing of this information through social networking applications. It automatically infers people's sensing presence (e.g., dancing at a party with friends) and then shares this presence through social network portals such as Facebook.

In contrast to the methods and applications mentioned above, the proposed framework in this paper illustrates a methodology to automatically detect the existence of types of device features and functionalities that the learner may use in his/her device for learning. It is not targeting a particular feature, or a certain device type or operation system. In addition, it monitors how the learner interacts with and uses each feature in order to understand and recognize the learner's preferences on how to use mobile devices for learning. Such context information opens up new possibilities for providing adaptive learning environment. 


\section{Framework}

The proposed framework (Fig. 1) is a client-server generic framework designed to run on smartphones, tablets, laptops and desktop-computers. On the client side - running on the learners' devices - the application is divided into two parts. The first one is the User Part, where personal information about the user (i.e., learner) is gathered and usage data visualization is presented. This part has two components: the Personal Information Manager, allowing the learner to login and to provide personal information (e.g., login name, full name, email address and other characteristics). This information is grouped under the name of User Object. The second component is the Visualizer, which is responsible for providing a user interface to show the feature availability and usage information. The second part of the application is the Device Part, where device information about the availability and usage of features is collected. This is done by Feature Detectors and the Tracker. Each Feature Detector is associated to a feature (e.g., internet connection types, keyboard presence, available touch screen) and able to discover whether the respective feature is available in that device. Table 1 presents all features currently considered in the framework. The data collection from all Feature Detectors is used to create the Device Object, which includes information to describe the tracked device in terms of its features. Furthermore, the Feature Detectors provide information about the usage of the respective features to the Tracker. The Tracker gathers the learner's device usage data by receiving updates from the Feature Detectors and stores this information on the server. However, if there is no connection to the server available, the Tracker creates a local backup file with the gathered data and postpones the online storage until connection to the server is available. Moreover, there is a component which belongs to both parts of the client side, the Detection Manager. The Detection Manager allows the learner to select what features the system should track. In order to do so, the Detection Manager detects what features are available in the device by calling the Feature Detectors when the learner starts the application for the first time and displays these features for selection. The set of information about the features selected by the learner is grouped under the name of Particular Device Object and represents a subset of the information in the Device Object. Furthermore, the Detection Manager encapsulates the user id, the device id and the Particular Device Object and sends all this information to the Tracker.

The server side mainly manages the database and the identification of devices and users, and is divided into two parts. Part A communicates with the User Part of the client and consists of the following components: the User Profiler, which is responsible for receiving the user information as a User Object from the client and for storing it in the database. The second component is the User Profile Structure, which stores what personal information of the learner is considered in the framework; and the third component is the XML Creator which processes the availability and usage data and generates a XML file which is used by the Visualizer. 


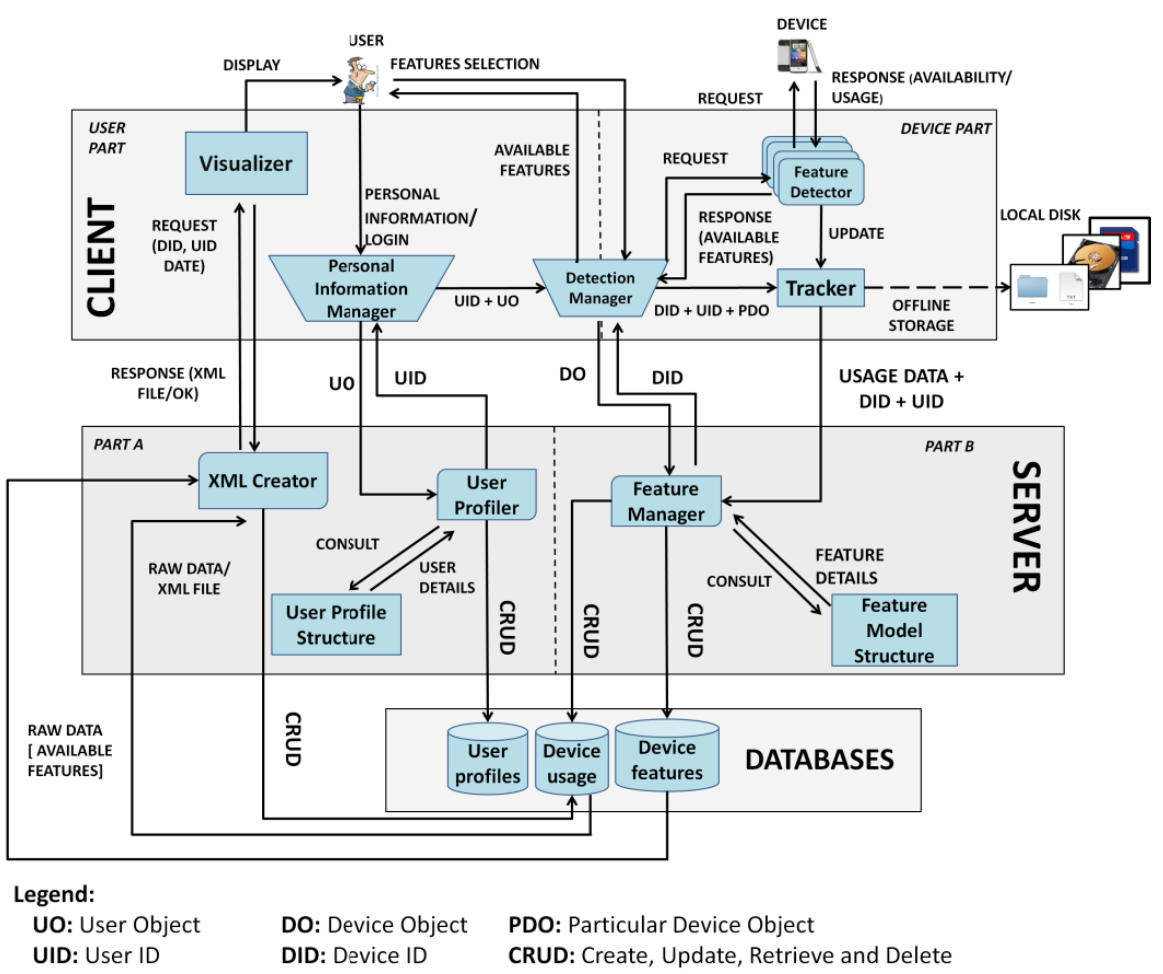

Fig. 1. The proposed framework architecture

Part B of the server side is responsible for the communication with the Device Part of the client. It is composed of the Feature Manager and the Feature Model Structure. The former is responsible for receiving the device information in form of a Device Object, including the available features on the device, and its usage data from the client, and for storing this information in the database. The Feature Model Structure describes the device features considered in the system. Moreover, there is a database, divided into three parts: First, it stores the device information, including the device id and which features are available to be used. In other words, it stores a set of Device Objects. Second, it stores user information (e.g., login name, full name, email address and other characteristics), in other words, a set of User Objects. Third, it combines the data relating a device to a learner and stores the usage data of the device by the learner. 
Table 1. List of the trackable features

\begin{tabular}{|c|c|c|}
\hline Category & Feature name & $\begin{array}{c}\text { Brief description } \\
\end{array}$ \\
\hline \multirow{4}{*}{ Communication } & Bluetooth & $\begin{array}{l}\text { Bluetooth radio functionality allows a } \\
\text { device to wirelessly exchange data with } \\
\text { other Bluetooth devices over short } \\
\text { distances. }\end{array}$ \\
\hline & Wi-Fi & $\begin{array}{l}\text { Wi-Fi uses radio waves to provide } \\
\text { wireless high-speed Internet and network } \\
\text { connections based on IEEE } 802.11 \\
\text { standards }\end{array}$ \\
\hline & Telephony & $\begin{array}{l}\text { Telephony provides communication over } \\
\text { distances using electrical signals. }\end{array}$ \\
\hline & SMS & $\begin{array}{l}\text { Short Message Service (SMS) uses } \\
\text { standardized communications protocols } \\
\text { that allow the exchange of short text } \\
\text { message. }\end{array}$ \\
\hline \multirow{2}{*}{ Location } & GPS & $\begin{array}{l}\text { Global Positioning System (GPS) is a } \\
\text { receiver of satellite based signal } \\
\text { information to identify the user's location } \\
\text { (outdoor). }\end{array}$ \\
\hline & Network Location & $\begin{array}{l}\text { It uses coarse location coordinates } \\
\text { obtained from a network-based } \\
\text { geolocation system to identify the user's } \\
\text { location. }\end{array}$ \\
\hline \multirow{8}{*}{ Sensors } & Camera & A camera is used to capture images. \\
\hline & Microphone & $\begin{array}{l}\text { A microphone converts sound into an } \\
\text { electrical signal. }\end{array}$ \\
\hline & Barometer & $\begin{array}{l}\text { A barometer is a tool for measuring } \\
\text { atmospheric pressure. }\end{array}$ \\
\hline & Compass & $\begin{array}{l}\text { A compass is a magnetometer, which } \\
\text { provides directional readings. }\end{array}$ \\
\hline & Gyroscope & $\begin{array}{l}\text { A Gyroscope is a tool for measuring or } \\
\text { maintaining orientation based on the } \\
\text { principles of angular momentum. }\end{array}$ \\
\hline & Light & A light sensor measures the light level. \\
\hline & Proximity & $\begin{array}{l}\text { A proximity sensor is used to measure } \\
\text { how close an object is to the device. }\end{array}$ \\
\hline & Accelerometer & $\begin{array}{l}\text { An accelerometer is a tool that } \\
\text { measures proper acceleration. }\end{array}$ \\
\hline \multirow{3}{*}{ Input } & Soft Keyboard & $\begin{array}{l}\text { A soft keyboard is an on-screen virtual } \\
\text { keyboard. }\end{array}$ \\
\hline & Hard Keyboard & $\begin{array}{l}\text { A hard keyboard is a real (hardware) } \\
\text { device. }\end{array}$ \\
\hline & Touchscreen & $\begin{array}{l}\text { A touchscreen is an electronic visual } \\
\text { display that allows the user to control the } \\
\text { device through simple or multi-touch } \\
\text { gestures. }\end{array}$ \\
\hline
\end{tabular}




\section{$4 \quad$ Implementation for Android Phones}

As mentioned above, the proposed framework is designed for tracking and analyzing the availability and usage of device features from different devices such as mobile phones, tablets and desktop-computers. In this section, we introduce an application for mobile phones, running on the Android operation system.

This application - the Usage Observer - aims at providing the possibility for learners to select features on their devices, monitor the usage of those features and view data about feature availability and usage in a user-friendly way. Moreover, learners can allow teachers to access these data. The application is composed of seven interfaces: login, main, feature selection, personal information/preferences, tracker management, feature usage visualization and administration. Some of the major interfaces can be seen in Fig. 2 and Fig. 3.

The login and registration process is managed by the Personal Information Manager and the Detection Manager. If the learner does not have an account yet, he/she needs to go through the registration process. In the first step, the learner can provide personal information and preferences (as shown in Fig. 2b), leading to the creation of a User Object by the Personal Information Manager. This interface (as well as the other user interfaces) is implemented as an Android component extension called Activity.

In the second step of the registration process, the learner registers his/her device by accessing the Detection Manager, where he/she can select which features should be tracked (Fig. 3a). This interface presents the device's available features, identified by the Feature Detectors, and allows the learner to select which features he/she wants to be tracked. As a result, a Particular Device Object is created containing information on those selected features. The Feature Detectors are implemented as one single service, called Detection Service. Services are an Android component extension, which run in the background and typically perform long-term tasks. If a learner is using different devices, the registration for the user account has to be done only once but whenever the learner is using a new device, this device has to be registered by performing the second step of the registration process. Once the learner and his/her device are registered, he/she can login by providing the correct username and password (Fig. 2a) and is presented with the main interface (Fig. 3b), where he/she can navigate through the application.

The main interface allows the learner to access the personal information entered in the registration process (through the Preferences symbol) and the feature selection interface (through the Features symbol) in order to change personal information and selected features. Furthermore, the main interface provides access to the tracking and visualization interfaces. The tracking interface presents raw data about the tracked features (accessible through the Tracking symbol) and the visualization interface shows user-friendly visualizations about the availability and usage of features on the learner's devices (accessible through the Visualize symbol). Administrators and teachers can additionally access the administrator interface (through the Admin symbol), which provides them with additional visualization interfaces to compare usage information from different learners and/or different devices. 


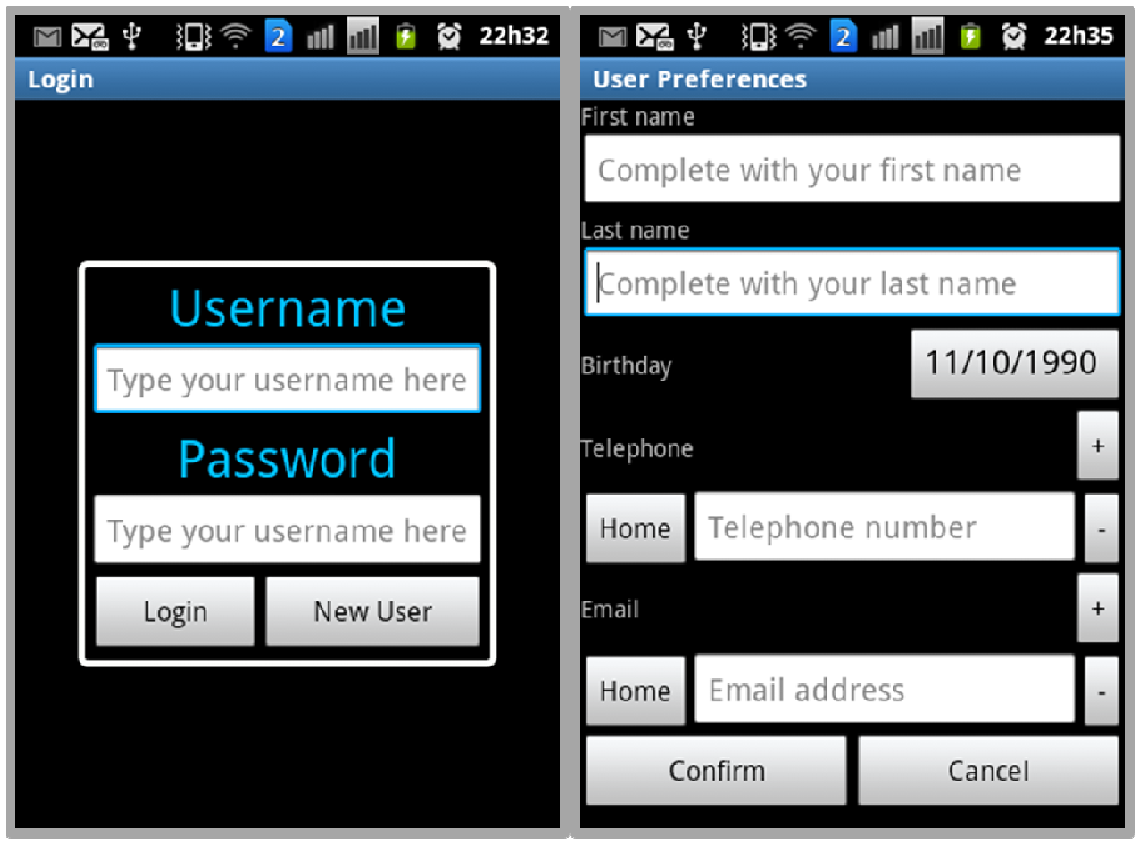

Fig. 2. Application interfaces $-2 a$ : Login interface; $2 b$ : Personal information interface

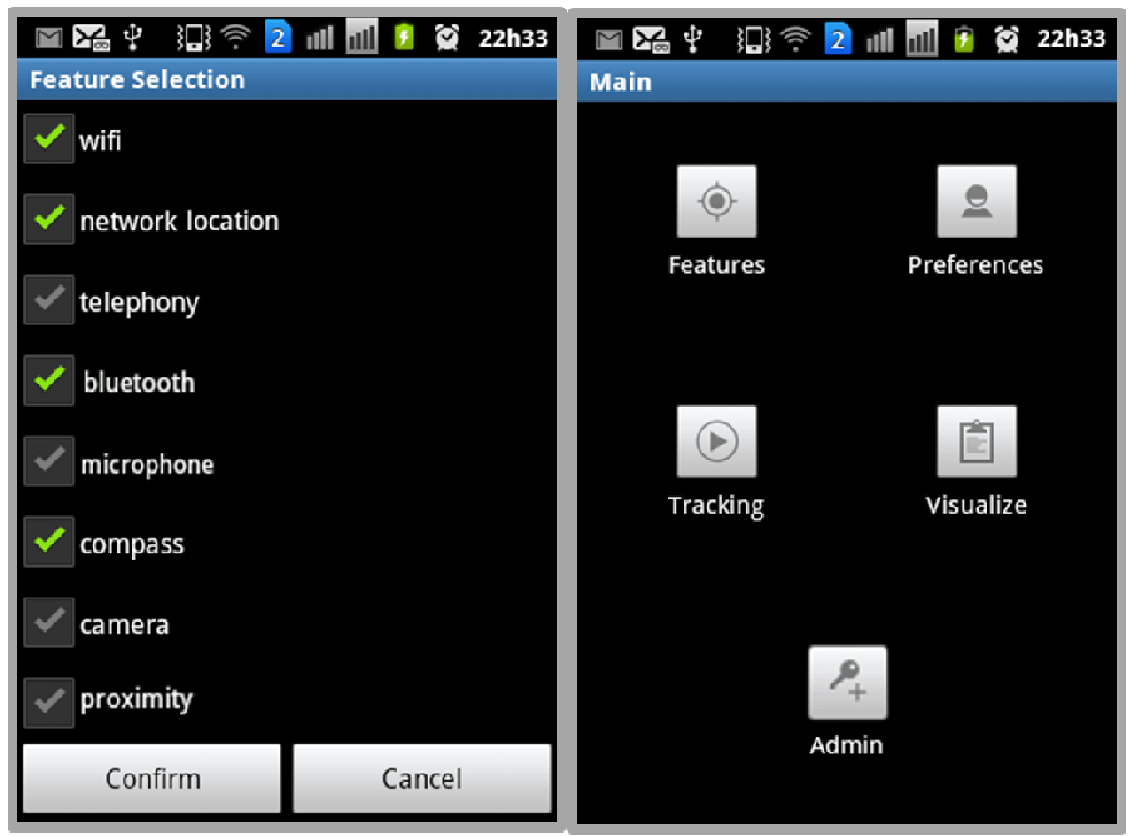

Fig. 3. Application interfaces - 3a: Feature selection interface; $3 b$ Main interface (access point of the other interfaces) 
While learners are using their devices, the system tracks the usage of the selected features. This is done by the Tracker Service, which represents part of the Feature Detectors and part of the Tracker component in the framework. The Tracking Service is responsible for collecting data about the usage of the previously selected features. One of the biggest development challenges was to implement the algorithm for the Tracking Service to track the usage of the selected features. The Android platform does not provide any direct way of collecting this type of data for certain features [22]. After evaluating several different approaches, we implemented this service by reading the system generated logs and gathering the timestamps related to the use of each selected feature. Hence, the Tracker component in the framework works as a logger which means the developed Tracker service collects log data provided by the system and sends it to the framework's server side. The server is then responsible for parsing the received log data and for obtaining the selected feature's usage data and statistics based on the timestamps contained in the log data. This task is performed by the Feature Manager component, which then stores the new information in the database.

\section{Conclusions and Future Work}

This paper introduces a framework that aims at building a comprehensive context profile through detecting available features of a device (e.g., keyboard, touch screen, internet connection, camera, GPS, and so on) and tracking the usage of these features by its users. Furthermore, it visualizes the gathered data in a user-friendly way. The proposed framework is designed for different devices such as smartphones, tablets and desktop-computers.

The gathered data about availability and usage of device features can be used in many ways to improve mobile learning and has high potential to help in enhancing learning outcomes of mobile learners. First, the gathered information is the basis for extending learning systems with advanced adaptive and intelligent capabilities that allow personalizing user interfaces and providing learners with adaptive course structures and recommendations based on availability and previous usage of device features. Second, the gathered data provides insights into what features are frequently available and used, which gives information about what kind of learning activities are most useful for facilitating mobile learning, as well as gives teachers feedback on how suitable their courses are for mobile learning. Third, the gathered data can be used to analyze successful learning with mobile and desktop-based devices and provide learners with personalized support and suggestions on how to improve their learning using different devices.

Based on the proposed framework, an application is implemented to run on Android devices. It is a client-server application which stores and analyzes the collected usage data on the server side. In order to keep the user's privacy, the application allows the learner to decide which features to track and when. In addition, the learner has the ability to delete his/her recent or entire usage history. In our future work, we plan to complete the development of the proposed framework, with focus on 
the server-side components and the visualization component. We also plan to implement applications for other devices such as tablets, desktop computers, other mobile phones, etc. Furthermore, we plan to perform an experiment to evaluate the system efficacy and usefulness for learners and teachers. Such evaluation will also help us in identify limitations of our system which can then be addressed in future work.

Acknowledgements. The authors acknowledge the support of NSERC, iCORE, Xerox, Mitacs, and the research-related gift funding by Mr. A. Markin.

\section{References}

1. Graf, S., MacCallum, K., Liu, T., Chang, M., Wen, D., Tan, Q., Dron, J., Lin, F., Chen, N., McGreal, R., Kinshuk, K.: An infrastructure for Developing Pervasive Learning Environments. In: IEEE International Workshop on Pervasive Learning, pp. 389-394. IEEE Press (2008)

2. Tortorella, R., Graf, S.: Personalized Mobile Learning Via An Adaptive Engine. In: IEEE International Conference on Advanced Learning Technologies, pp. 670-671. IEEE Press (2012)

3. Chen, G.D., Chang, C.K., Wang, C.Y.: Ubiquitous learning website: Scaffold learners by mobile devices with information-aware techniques. Computers \& Education 50(1), 77-90 (2008)

4. Schmidt, A.: Implicit Human Computer Interaction Through Context. Personal Technologies 4(2\&3), 191-199 (2000)

5. Ziefle, M., Himmel, S., Holzinger, A.: How usage context shapes evaluation and adoption criteria in different technologies. In: International Conference on Applied Human Factors and Ergonomics, San Francisco, pp. 2812-2821 (2012)

6. Ogata, H., Li, M., Hou, B., Uosaki, N., El-Bishouty, M.M., Yano, Y.: SCROLL: Supporting to Share and Reuse Ubiquitous Learning Log in the Context of Language Learning. In: World Conference on Mobile and Contextual Learning, pp. 40-47 (2010)

7. Roman, M., Campbell, R.H.: A User-Centric, Resource-Aware, Context-Sensitive, MultiDevice Application Framework for Ubiquitous Computing Environments. Technical Report (2002),

http: / / gaia.cs.uiuc . edu/papers / new080405/AppFramework1. doc (accessed on April 15, 2013)

8. PhoneUsage, https://play.google.com/store/apps/

details?id=com. jupiterapps . phoneusage\&hl=en

(accessed on April 15, 2013)

9. Elixir, https://play.google.com/store/apps / details?id=bt. android.elixir (accessed on April 15, 2013)

10. Smith, S.D., Salaway, G., Caruso, J.B.: The ECAR study of undergraduate students and information technology. EDUCAUSE Center for Applied Research (2009), http: / / www . educause. edu/ library/ERS0906 (accessed on April 15, 2013)

11. ECAR study of undergraduate students and information technology,

http: / / www. educause.edu/library/resources /

ecar-study-undergraduate-students-and-information-

technology-2012 (accessed on April 15, 2013) 
12. Oliver, B., Nikoletatos, P.: Building engaging physical and virtual learning spaces: A case study of a collaborative approach. In: Same Places, Different Spaces, The Annual Australian Society for Computers in Learning in Tertiary Education Conference, pp. 720-728 (2009)

13. Oliver, B., Whelan, B.: Designing an e-portfolio for assurance of learning focusing on adoptability and learning analytics. Australasian Journal of Educational Technology 27(6), 1026-1041 (2011)

14. Ally, M.,Palalas, A.:State of Mobile Learning in Canada and Future Directions (2011), http: / /www.rogersbizresources.com/files/308/

Mobile_Learning_in_Canada_Final_Report_EN.pdf (accessed on April 15, 2013)

15. Kennedy, G.E., Judd, T.S., Churchward, A., Gray, K., Krause, K.-L.: First year students' experiences with technology: Are they really digital natives? Australasian Journal of Educational Technology 24(1), 108-122 (2008)

16. Algonquin College,a new era of connectivity at Algonquin College: Collaborative approach to Mobile Learning Centre, a first in Canada, http: / /www.algonquincollege.com/PublicRelations/Media/

$2011 /$ Releases/MobileLearningCentreNewsRelease.pdf (accessed on April 15, 2013)

17. Trinder, J.J.:Mobile learning evaluation: the development of tools and techniques for the evaluation of learning exploiting mobile devices through the analysis of automatically collected usage logs - an iterative approach, $\mathrm{PhD}$ thesis (2012), http: //theses.gla.ac.uk/3303/(accessed on April 15, 2013)

18. Trinder, J.J., Magill, J.V., Roy, S.: Using automatic logging to collect information on mobile device usage for learning. In: Pachler, Kukulska-Hulme, Vavoula (eds.) Research Methods in Mobile and Informal Learning. Peter Lang Publishing Group (2009)

19. 35 Android Apps to Monitor Usage Stats and Tweak System Utilities, http: //android.appstorm. net/roundups / utilities-roundups / 35-android-apps-to-monitor-usage-statsand-tweak-system-utilities / (accessed on April 15, 2013)

20. Android Status, https://play.google.com/store/apps/developer?id=androidstatus (accessed on April 15, 2013)

21. Miluzzo, E., Lane, N., Fodor, K., Peterson, R., Lu, H., Musolesi, M., Eisenman, S., Zheng, X., Campbell, A.: Sensing Meets Mobile Social Networks: The Design, Implementation and Evaluation of the CenceMe Application. In: 6th ACM Conference on Embedded Network Sensor Systems, pp. 337-350 (2008)

22. Sensors Overview, http: / / developer.android.com/guide/topics/sensors / sensors_overview.html (accessed on April 15, 2013) 